in vivo $35: 325-332(2021)$

doi:10.21873/invivo.12263

\title{
Culture Separation, Identification and Unique Anti-pathogenic Fungi Capacity of Endophytic Fungi from Gucheng Salvia Miltiorrhiza
}

\author{
GUO-KAI WANG $^{1,2}$, JAI-SING YANG ${ }^{3}$, YU-FEI HUANG ${ }^{1}$, JIN-SONG LIU ${ }^{1,2}$, \\ CHIA-WEN TSAI ${ }^{3}$, DA-TIAN BAU ${ }^{3,4 *}$ and WEN-SHIN CHANG ${ }^{3 *}$ \\ ${ }^{1}$ School of Pharmacy, Anhui University of Chinese Medicine, Hefei, P.R. China; \\ ${ }^{2}$ Anhui Province Key Laboratory of Research and Development of Chinese Medicine, Hefei, P.R. China; \\ ${ }^{3}$ Department of Medical Research, China Medical University Hospital, \\ China Medical University, Taichung, Taiwan, R.O.C.; \\ ${ }^{4}$ Department of Bioinformatics and Medical Engineering, Asia University, Taichung, Taiwan, R.O.C.
}

\begin{abstract}
Background: Salvia miltiorrhiza is a medical herb for human disorders including cardiovascular diseases and cancer. However, the interactions between Salvia miltiorrhiza and its endophytes are largely unknown. The current study aimed at identifying its endophytic fungi and examining their inhibitory effects on anti-pathogenic fungus. Materials and Methods: Distinct species of endophytic fungi were isolated from the roots of Salvia miltiorrhiza, cultured, sequenced, aiming to predict their taxonomical structures. Meanwhile, extracts from each endophytic fungus fermentations were isolated, compared and evaluated on the inhibitory efficacies on five pathological fungi, Cercospora nicotianae, Phoma arachnidicola, Staphylococcus, Phytophthora eggplant, and Rhizoctonia cerealis. Results: A total of 34 strains of endophytic fungi were obtained from Salvia miltiorrhiza. Among them, SX19 and C. Gloeosporioids exhibited the most effective inhibitions on five pathogenic fungi. Conclusion: The anti-fungal activities of the endophytic fungus from Salvia miltiorrhiza were confirmed for the first time, and this may benefit crop quality and production in the future.
\end{abstract}

This article is freely accessible online.

*These Authors contributed equally to this study.

Correspondence to: Da-Tian Bau and Wen-Shin Chang, Terry Fox Cancer Research Laboratory, Department of Medical Research, China Medical University Hospital, 2 Yuh-Der Road, Taichung, 404 Taiwan, R.O.C. Tel: +886 422053366 (Ext. 5805), e-mail: datian@mail.cmuh.org.tw; artbau2@gmail.com

Key Words: Anti-pathogenic fungi activity, endophytic fungi, pathogenic fungi, Salvia miltiorrhiza.
Endophytic fungi refer to those fungi which colonize inside the tissues of the host plants, typically causing no apparent symptoms to the hosts. They play mutually beneficial roles to medical plants, balance the plant micro-ecosystems, and have the capacity to produce some natural bioactive products such as secondary metabolites in the host plants (1-4). Noticeably, there are also some pathogenic fungi which are parasitic on plants and may cause various plant diseases. Up to $70 \sim 80 \%$ of crop diseases are caused by these pathogenic fungi, and there are several or even dozens of fungal diseases in every crop. From the era of 2000s, mounting evidence for endophytic fungi serving as protectors for host plants against these pathogenic fungi have been accumulated at a relatively slow speed and the literature is extremely limited (5-18). The screening methodology for endophytic fungi as potent antipathogenic fungicides is also developed (19-21), and very exciting, the subtle alterations are also getting started to be revealed at not only signaling interaction network but DNA and RNA levels systematically $(22,23)$.

For more than one thousand years, Salvia miltiorrhiza (also have the names of Salvia miltiorrhiza Bge, Salvia miltiorrhiza Bunge and Danshen, hereon abbreviated as SM) has been applied as a traditional Chinese herbs with several physiological effects such as activating blood circulation, easing the stasis pain, and strengthening the capacity of kidney as diuresis (24). With the development of Chinese medical science, the evidence between SM components and their efficacies to human diseases have been revealed in recent years. In brief, the active medical ingredients of SM are mainly divided into two groups, the hydrophilic phenolic acids and lipid-soluble tanshinones. The former group include salvianolic acid A (Sal A), salvianolic acid B (Sal B), lithospermic acid, rosmarinic acid, and danshensu $(25,26)$, while the latter group include tanshinone I, tanshinone IIA, 
tanshinone IIB, cryptotanshinone, and dihydrotanshinone I (27). Take Salvianolic acids, which are found to be most abundant SM compounds, for example, they are reported to have beneficial capacities including anti-inflammatory (28), antioxidant (29), anti-thrombotic (30), and cardio-protective $(31,32)$ efficacies. Many laboratories report that extracts of SM dried roots may have therapeutic effects for a panel of human diseases, including several types of cancers $(33,34)$, cardiovascular diseases (34-37), neuronal disorders (34, 38), Alzheimer's disease (39) and liver diseases $(34,40)$. A stateof-arts summary about the signaling network of SM in its therapeutic affects was proposed by Jung and his colleagues in 2020 (34). However, the knowledge on endophytic fungi is relatively limited. In 2015, Li and his colleagues firstly isolated endophytic fungi from SM and examined their individual antioxidant capacity (41). They concluded that the antioxidant potential positively correlated to the phenolic and flavonoid compounds present in the ethanol extracts of A. alternata SaF-2 and F. proliferatum SaR-2 from SM (41). In 2016, another group found that a specific endophytic fungi of SM, Phoma glomerata D14, is capable of producing salvianolic acid C (42).

Up to now, there is no study identifying the components of MS endophytic fungi and examining their anti-fungal capacities on pathogenic fungi. Geographically, SM is mainly produced in Anhui and Jiangsu provinces of China, while the SM produced in Gucheng (the county area named as "ancient city") characterized with red-colored skin, purple and grainy meat, dry and loose quality, bid head without reed, is uniquely famous for its therapeutic application. Based on the above information, we aimed to systematically isolate the endophytic fungi from Gucheng SM roots, identify and classify them from a genetic point of view, and investigate their overall anti-fungal capacities in a panel of pathogenic fungi.

\section{Materials and Methods}

Isolation and morphological identification of endophytic fungi from Gucheng SM. Gucheng SM was collected from Anhui Hanshan Zhongda Shennong Co., Ltd, in July of 2017, and identified by Nian-Jun Yu of the Anhui University of Chinese Medicine. Briefly, fresh and healthy SM was surface sterilized as follows: samples were washed in $75 \%$ ethanol for $3 \mathrm{~min}$, followed by rinsing with autoclaved distilled water, then immersed in $3 \%$ sodium hypochlorite for $3 \mathrm{~min}$, rinsed with autoclaved distilled water, and finally rinsed with $75 \%$ ethanol again for $30 \mathrm{~s}$. The effective surface sterilization was ensured via inoculating the final wash onto potato dextrose agar (PDA, Beijing Aobox Biotechnology, PR China) and $\mathrm{LB}$ plates at $25^{\circ} \mathrm{C}$ and $37^{\circ} \mathrm{C}$, respectively. The results showed that all the washes had no growth. The sterile filter paper is blotted dry and the sterile blade was cut into small pieces. The tissue pieces were inoculated on the medium and cultured in a constant temperature incubator for 5-15 d. The grown hyphae were transferred onto new PDA plates with tip hyphae picking, grown to almost full plate, and repeat the processes of tip hyphae picking and re-grown for thrice for the pure single colony. Then, three full plates with individual fungus were ready for fungus inhibitory tests.

Genetic and taxonomic identification of endophytic fungi. Genomic DNA from cultured fungal endophytes was extracted using the plant/fungal genome DNA Mini-Preps Kit (Tiangen Biotech Co. Ltd., Beijing, PR China) and immediately quantified using a Nanodrop machine (Thermo Scientific, Waltham, MA, USA). The extracted DNA served as template for PCR amplify of Internal Transcribed Spacer (ITS) with the forward primer rDNA-ITS (5'TCCGTAGGTGAACCTGCGG-3') together with primer ITS4 (5'TCCTCCGCTTATTGATATGC-3' and primer ITS1 (5'-TCCGGTG AACCTGCGG-3'), respectively. The PCR amplification conditions were set as: initial denaturation for $5 \mathrm{~min}$ at $95^{\circ} \mathrm{C}$, followed by 35 amplification cycles $\left(95^{\circ} \mathrm{C}\right.$ for $30 \mathrm{~s}, 49^{\circ} \mathrm{C}$ for $30 \mathrm{~s}, 72^{\circ} \mathrm{C}$ for $90 \mathrm{~s}$ ), and a final extension at $72^{\circ} \mathrm{C}$ for $7 \mathrm{~min}$, using a Biometra PCR thermal cycler. The PCR products were separated with a $2 \%$ agarose gel electrophoresis at 100 Volts; the bands were excised and eluted using a gel purification kit (Illustra GFX96 PCR Purification kit, GE Healthcare, Chicago, IL, USA). The purified DNA was sequenced and subject to the sequence comparisons using BLAST searches to Genbank on NCBI website. The phylogenetic trees were constructed according to the Kimura2-Parameter model with Neighbor-Joining cluster analysis using MEGA ver. 5.0. System evolutionary matrix estimation, and each train was identified by the stability of the tree topology with 1,000 cycles.

Preparation of endophytic fungal fermentation products. The endophytic fungi of Gucheng SM were inoculated into PDA medium and cultured at $28^{\circ} \mathrm{C}$ for 5-7 days in the dark, and it was punched with a puncher of about $6 \mathrm{~mm}$. Water was added in the potato dextrose broth medium powder, mixed well, sterilized for $20 \mathrm{~min}$, cooled, and picked up of 2 or 3 pieces for perforated endophytic fungus tablets and place them in a liquid medium. The shaker was set as $180 \mathrm{rpm}$, and the fermentation was carried out at $28^{\circ} \mathrm{C}$ for $7 \mathrm{~d}$. After filtration, the fermentation broth was extracted with ethyl acetate, concentrated and evaporated to dryness. A total of $1 \mathrm{mg} / \mathrm{ml}$ solution was prepared in sterile water, filtered, and set aside.

Anti-pathologic fungi screening procedure. Cercospora nicotianae, Phoma arachnidicola, Staphylococcus, Phytophthora eggplant, and Rhizoctonia cerealis were kindly offered by Dr. Bin-Ji Ma of Henan Agricultural University. The five species of pathogenic fungi are the components of our anti-fungal screening platform.

Each pathogenic fungus was inoculated into PDA medium, and then transferred to a new medium, and cultured at $26^{\circ} \mathrm{C}$ for 5 days at constant temperature along the edge of the colony. These were captured into $6 \mathrm{~mm}$ pieces by a puncher. It contained $1 \mathrm{ml}$ of fermentation broth for each endophyte in each medium plate with $10 \mathrm{ml}$ medium. The pathogenic fungi were inoculated into them, and the rate of anti-pathogenic fungi capacity is determined by a cross method. The agar plates were incubated at $30^{\circ} \mathrm{C}$ for $48 \mathrm{~h}$ in darkness. The radius of each zone of inhibition was measured. The combined fungicides Amphotericin B (Catalog\#A2942, Sigma Aldrich, St. Louis, Mo, USA) and Nystatin (Catalog\#N6261, Sigma Aldrich) were used as the positive control, and distilled water was used as a negative control. Each endophyte was screened in three independent replicates in the first turn, and Each endophyte were overall re-screened for activity against the five pathogenic fungi 
Table I. Compositions and diversities of endophytic fungi in Gucheng Salvia miltiorrhiza.

\begin{tabular}{|c|c|c|c|c|c|}
\hline Genus & Species & No. & GenBank accession number & Similarity & No. of strains \\
\hline \multirow[t]{4}{*}{ Aspergillus } & Flavus & DS-5 & LN482516.1 & 99 & 2 \\
\hline & Ochrceus & DS-6 & KU743890.1 & 99 & 4 \\
\hline & Niger & DS-7 & KU904480.1 & 99 & 3 \\
\hline & Udagawae & DS-12 & KY808744.1 & 99 & 2 \\
\hline \multirow[t]{3}{*}{ Fusarium } & Proliferatum & DS-3 & MG543763.11 & 100 & 2 \\
\hline & $S p . R 24$ & DS-9 & KM979804.1 & 99 & 2 \\
\hline & Verticillioides & DS-10 & КC684889.1 & 99 & 1 \\
\hline \multirow[t]{2}{*}{ Epicoccum } & Sp. SX19 & DS-4 & КC329632.1 & 99 & 1 \\
\hline & Nigrum & DS-13 & MF800916.1 & 99 & 2 \\
\hline Arthrinium & Sp. LH11 & DS-1 & HQ832842.1 & 99 & 3 \\
\hline Coprinellus & Radians & DS-2 & FJ462761.1 & 99 & 2 \\
\hline Dictyosporium & Aquaticum & DS-8 & KM610236.1 & 95 & 3 \\
\hline Colletotrichum & Gloeosporioides & DS-11 & КР900290.1 & 100 & 3 \\
\hline Rhizoctonia & Bataticola & DS-14 & HQ392782.1 & 99 & 2 \\
\hline Phomopsis & $T P-41$ & DS-15 & DQ780435.1 & 98 & 1 \\
\hline Pithomyces & Chartarum & DS- 16 & GU073119.1 & 99 & 1 \\
\hline
\end{tabular}

again in triplicate to confirm the antifungal activity spectrum for each extract.

Statistical and evaluation system. Inhibitory rate (\%) is calculated with the formula: (control plate colony diameter - fermentation broth colony diameter)/control plate colony diameter $\times 100 \%$. All statistical analysis had been conducted using Excel. All data shown in the Table II represent the average of six replicates.

\section{Results}

Morphological identification results of endophytic fungi. A total of 34 endophytic fungi were obtained from SM, belonging to 10 genera and 16 species (named as DS-1 to DS-16), mainly distributed in Fusarium, Epicoccum, and Aspergillus (Table I). The characteristics of the endophytic fungal colonies obtained were isolated according to the "Handbook of identification of fungi", such as edge shape, hypha color, transparency, surface wet and dry state, shape, size and other characteristics (43). According to the typical classification of fungal morphology (44), each endophytic fungus was microscopically determined. Figure 1 shows each endophytic fungus in $1 \times$ and $40 \times$ under microscope.

In detail, the DS-5, DS-6, DS-7, DS-12 are of the common characters including conidia, spherical, elliptical or pearshaped, light yellow or yellow colored, easy to scatter, spores brownish green in microscopic, irregular aggregation distribution, all belong to the genus Aspergillus (Table I, top genus). The DS-3, DS-9, and DS-10 have the following characteristics: white, relatively dense and flocculent surface hyphae, conidia existed in large numbers, wedge-shaped or fusiform file type, with the obvious diaphragm, 3 to 6 membranes (most of them are 3 and several are 4-6). They are all identified as the genus Fusarium (Table I, second genus).
The sporodochium of DS-4 are dark-colored and cushionshaped, while their conidiophores are short and dark-colored. Their spores are globose and dark-colored and stored in irregularly brick-wall-like structures. Like those of DS-4, the spores of DS-13 are also globose and dark-colored. However, the spores of DS-13 are characterized with verrucous protrusions and wrinkled rugoses, and relatively lower infection activities. The DS-1 is of branched filamentous body, no septum, a split fruit production type, and a thick spore formed in the hyphae, and identified as the family Arthrinium. The DS-2 is produced on the pleats or in the pores. The fruiting body has a stalk and was born in the center of the cap. It is identified as Coprinellus fungus. The spores of DS-8 are stored in brick-wall-like structures and are almost colorless. The DS-11 that has a surface that is hyphae gray-brown, dense, blister-like, conidial stalk single, unbranched, bristles black, sparse, perennial, microscopic hyphae, radial distribution, was identified as Colletotrichum. The diameters of sclerotium for DS-14 can vary from 5 to $100 \mu \mathrm{m}$, while their conidiophores are cylindrical with elliptical or clavarialike micro-bending spores. The DS-15 has spherical of conidia, oval or stick-shaped, leathery, dark brown, cracked by small holes, epigenetic, conidial single cavity, born on the surface of the sub-seat, identified as Phomopsis Fungus. The conidiophore and spores of DS-16 are colorless, while spores are multi-membraned with various shapes including fusiform, elliptical, cylindrical and globose.

Genetic identification and phylogenetic tree of Gucheng SM endophytic fungi. The DNA of endophytic fungi were amplified by rDNA-ITS methodology, and the PCR amplicons for ITS domain of each strain purified, was sequenced and 
in vivo $35: 325-332(2021)$

Table II. Inhibitory effects for 14 endophytic fungi of seven dominant species in Gucheng Salvia miltiorrhiza on 5 plant pathogenic fungi.

\begin{tabular}{|c|c|c|c|c|c|}
\hline \multirow[t]{2}{*}{ Endophytic fungus } & \multicolumn{5}{|c|}{ Inhibitory rate for individual pathogenic fungus (\%) } \\
\hline & Cercospora nicotianae & Phoma arachnidicola & Staphylococcus & Phytophthora eggplant & Rhizoctonia cerealis \\
\hline E.sp. SX19 & $60.3 \pm 2.2 \%$ & $57.6 \pm 8.1 \%$ & $58.6 \pm 6.6 \%$ & $68.6 \pm 6.6 \%$ & $38.5 \pm 3.7 \%$ \\
\hline A.Ochrceu & $38.0 \pm 8.2 \%$ & $26.1 \pm 9.7 \%$ & $54.1 \pm 3.8 \%$ & $46.5 \pm 5.7 \%$ & $45.9 \pm 7.8 \%$ \\
\hline A.niger & $21.8 \pm 3.5 \%$ & $2.5 \pm 1.4 \%$ & $44.5 \pm 3.0 \%$ & $54.5 \pm 7.1 \%$ & $53.7 \pm 2.0 \%$ \\
\hline F.sp. R24 & $18.9 \pm 2.9 \%$ & $7.5 \pm 5.0 \%$ & $34.8 \pm 9.9 \%$ & $55.5 \pm 4.1 \%$ & $44.5 \pm 1.0 \%$ \\
\hline F.verticillioide & $21.2 \pm 9.7 \%$ & $24.4 \pm 1.3 \%$ & $41.1 \pm 7.6 \%$ & $48.4 \pm 9.5 \%$ & $53.2 \pm 4.0 \%$ \\
\hline A.udagawae & $23.3 \pm 10.8 \%$ & $10.1 \pm 1.3 \%$ & $41.9 \pm 8.0 \%$ & $57.2 \pm 4.9 \%$ & $48.5 \pm 7.0 \%$ \\
\hline E.nigrum & $15.7 \pm 5.6 \%$ & $5.7 \pm 3.1 \%$ & $18.4 \pm 3.8 \%$ & $54.5 \pm 4.3 \%$ & $48.0 \pm 8.5 \%$ \\
\hline A.sp. LH11 & $23.1 \pm 8.2 \%$ & $20.5 \pm 5.1 \%$ & $36.0 \pm 5.5 \%$ & $25.7 \pm 2.9 \%$ & $17.3 \pm 3.8 \%$ \\
\hline C.radians & $25.1 \pm 4.2 \%$ & $27.2 \pm 3.8 \%$ & $34.1 \pm 1.1 \%$ & $30.7 \pm 3.9 \%$ & $27.1 \pm 2.3 \%$ \\
\hline F.proliferatum & $25.1 \pm 6.6 \%$ & $26.2 \pm 10.3 \%$ & $38.3 \pm 5.0 \%$ & $29.3 \pm 2.7 \%$ & $25.4 \pm 2.1 \%$ \\
\hline D.aquaticum & $23.5 \pm 3.2 \%$ & $27.7 \pm 6.5 \%$ & $30.0 \pm 5.9 \%$ & $23.1 \pm 5.8 \%$ & $24.2 \pm 2.5 \%$ \\
\hline C.Gloeosporioids & $49.6 \pm 5.0 \%$ & $62.1 \pm 5.2 \%$ & $61.9 \pm 3.5 \%$ & $51.1 \pm 6.2 \%$ & $54.5 \pm 4.2 \%$ \\
\hline P.TP-41 & $33.8 \pm 7.9 \%$ & $31.9 \pm 2.0 \%$ & $33.1 \pm 2.5 \%$ & $26.4 \pm 4.7 \%$ & $23.1 \pm 0.8 \%$ \\
\hline P.chartarum & $26.8 \pm 3.6 \%$ & $25.3 \pm 3.6 \%$ & $35.4 \pm 3.1 \%$ & $25.7 \pm 5.1 \%$ & $22.6 \pm 1.6 \%$ \\
\hline
\end{tabular}

E.sp. SX19: Epicoccum Sp. SX19; A.Ochrceu: Aspergillus Ochrceus; A.niger: Aspergillus Niger; F.sp. R24: Fusarium Sp. R24; F.verticillioide: Fusarium Verticillioides; A.udagawae: Aspergillus Udagawae; E.nigrum: Epicoccum Nigrum; A.sp. LH11: Arthrinium Sp. LH11; C.radians: Coprinellus Radians; F.proliferatum: Fusarium Proliferatum; D.aquaticum: Dictyosporium Aquaticum; C.Gloeosporioids: Colletotrichum Gloeosporioides; P.TP-41: Phomopsis TP-41; P.chartarum: Pithomyces Chartarum.

compared with each other for similarity. Finally, the phylogenetic trees for the Gucheng SM endophytic fungi are firstly constructed and shown in Figure 2. Noticeably, the diversities of Gucheng SM endophytic fungi are very high.

In vitro measurements of anti-pathogenic fungi activity. The inhibitory capacity against Cercospora nicotianae, Phoma arachnidicola, Staphylococcus, Phytophthora eggplant and Rhizoctonia cerealis for fermentation products of from the 14 endophytic fungi of seven dominant species in Gucheng Salvia miltiorrhiza were measured and summarized in Table II. Among the most effective endophytic fungi against pathogenic Cercospora nicotianae, Phoma arachnidicola and Staphylococcus, E.sp. SX19 and C. Gloeosporioids were the top two. As for Phytophthora eggplant, E.sp. SX19 was the top one and $C$. Gloeosporioids was the sixth with an inhibitory rate of $51.1 \%$. As for Rhizoctonia cerealis, C. Gloeosporioids was the top one and E.sp. SX19 was the eighth with an inhibitory rate of $38.5 \%$. Overall, the average inhibition rate of the fermentation products of $C$. Gloeosporioids against five crop pathogenic fungi were $56.72 \%$, and the average inhibition rate of E.sp. SX19 was $55.84 \%$ (Figure 3). The overall results show that these two endophytic fungi of Gucheng SM have good antibacterial effects on five pathogenic fungi.

\section{Discussion}

SM is a medicinal herb that has been applied in therapeutic treatments of various diseases such as cancer, cardiovascular diseases, liver diseases, nervous system diseases in East Asia. The endophytic fungi from SM may benefit the SM with antioxidant capacity (41), and elevating the production of salvianolic acid (42). However, the knowledge about their diverse species, taxonomic relationships, and anti-fungal capacities are extremely limited. In the current study, we have systematically cultured, isolated, and examined the endophytic fungi of Gucheng SM of their antifungal capacities against five crop pathogenic fungi, Cercospora nicotianae, Phoma arachnidicola, Staphylococcus, Phytophthora eggplant, and Rhizoctonia cerealis.

We identified up to 34 endophytic fungi from Gucheng GM, which belong to 10 genera and 16 species that are mainly distributed in Fusarium, Epicoccum, and Aspergillus (Table I). One of the highlight findings in the current study is that two of the strains of Gucheng SM endophytic fungi, E.sp.SX19 and C. Gloeosporioids, are of the high-inhibitory and broad-spectrum anti-fungal characters to effectively kill the fungus (Table II). In detail, the inhibitory capacities of E.sp. SX19 against Cercospora nicotianae, Phoma arachnidicola, Staphylococcus, Phytophthora eggplant, and Rhizoctonia cerealis are $60.3 \%$ (top of 14 examined), $57.6 \%$ $(2 / 14), 58.6 \%(2 / 14), 68.6 \%(1 / 14)$ and $38.5 \%(8 / 14)$, respectively. Those for $C$. Gloeosporioids are $49.6 \%(2 / 14)$, $62.1 \%(1 / 14), 61.9 \%(1 / 14), 51.1(6 / 14)$ and $54.5 \%(1 / 14)$, respectively. It is of great agricultural value to elevate the overall yield and quality of Gucheng SM via application of endophytic fungi such as E.sp. SX19 plus C. Gloeosporioids from lab to field. 


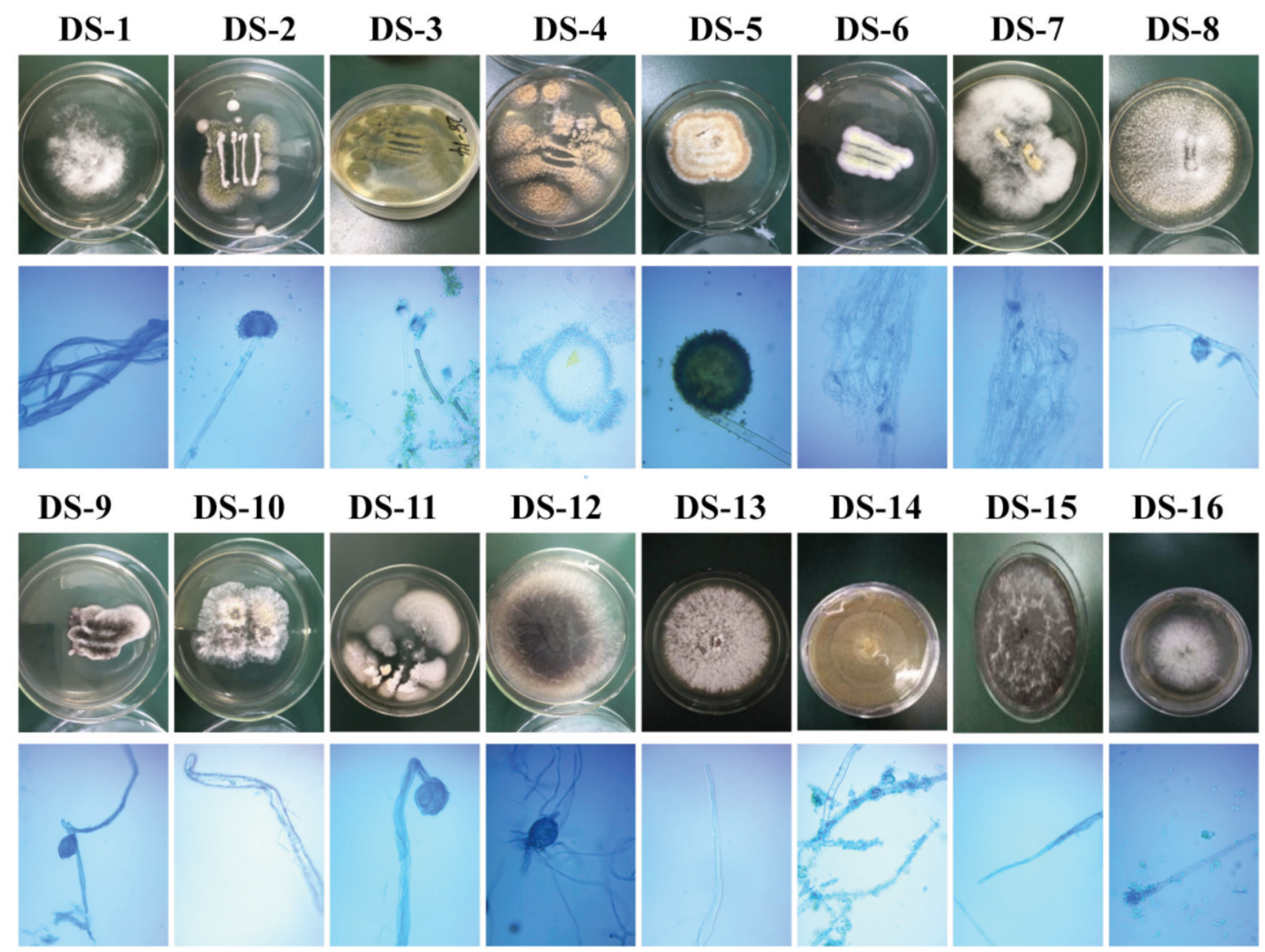

Figure 1. Colony and microstructure of DS1 16 endophytic fungi. Magnification $\times 1$ (upper panel) and $\times 40$ (lower panel) pictures of DS1 16 endophytic fungi taken under microscopy observation.

Several limitations of the study should be noted. First, the study confirmed the endophytic behaviors of fungal strains isolated to be beneficial for the host plants, further studies about the effective chemicals and detailed mechanisms are needed. Second, only ITS4 sequences were used for taxonomic identification of the putative fungal endophytes. Additional sequences such as $18 \mathrm{~S}$ will be required for further validation. Third, it is currently impossible to purify sufficient endophytic chemicals for in planta validation experiments, such as on host SM itself since we do not have their mechanisms clear enough. Potentially, the crude products from candidate endophytes could be subject to further identification of pure chemicals, and large-scale synthesis and semi-synthesis.

\section{Conclusion}

This is the first systematic investigation of SM endophytes of their antifungal capacity. To sum up, these endophytes showed inhibitory capacities against diverse pathogenic fungi, including Cercospora nicotianae, Phoma arachnidicola,
Staphylococcus, Phytophthora eggplant, and Rhizoctonia cerealis. Among them, E.sp. SX19 and C. Gloeosporioids have the highest potential in multiple suppression of pathogenic fungi, which will elevate the yield and quality of therapeutic medical crops, Gucheng SM. The antifungal capacities of Gucheng SM endophytes identified in this study will be of value in agricultural and clinical practice after further in planta experiments in the near future.

\section{Conflicts of Interest}

The Authors declare that no potential conflicts of interest exist.

\section{Author's Contributions}

Drs. Da-Tian Bau, Jai-Sing Yang and Guo-Kai Wang designed the overall research; Yu-Fei Huang and Jin-Song Liu performed the genetic and experimental research; Chia-Wen Tsai and Wen-Shin Chang contributed to the establishment and development of statistical and analyzing platform, while Guo-Kai Wang, Da-Tian Bau and Wen-Shin Chang contributed to the completing of the article. All Authors read and approved the final manuscript. 


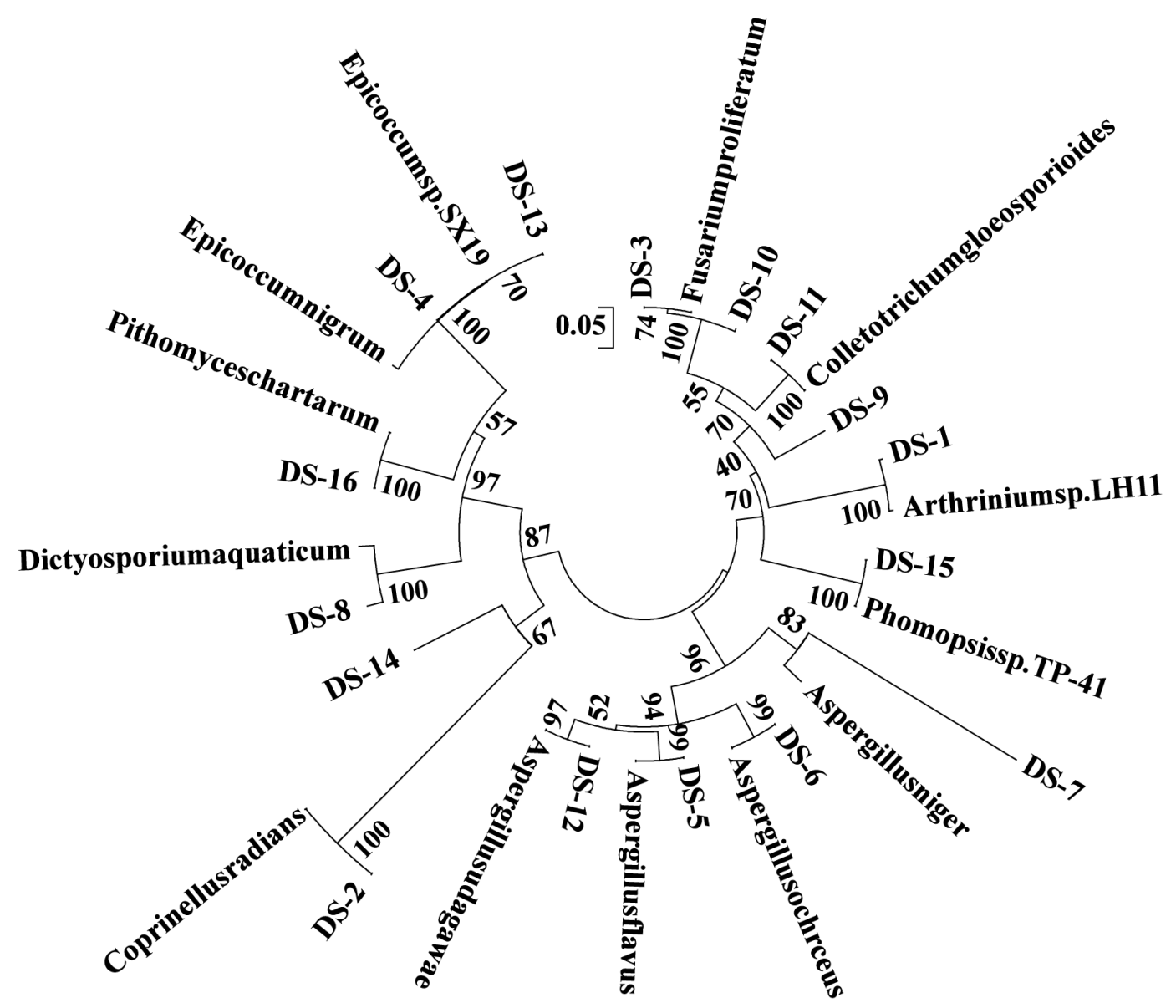

Figure 2. The phylogenetic tree of endophytic fungi based on ITS sequences. Each PCR product was excised and eluted from the agarose gel, while the purified DNA was sequenced and compared to Genbank on NCBI website. The phylogenetic trees were then constructed according to the Kimura2-Parameter model with Neighbor-Joining cluster analysis.

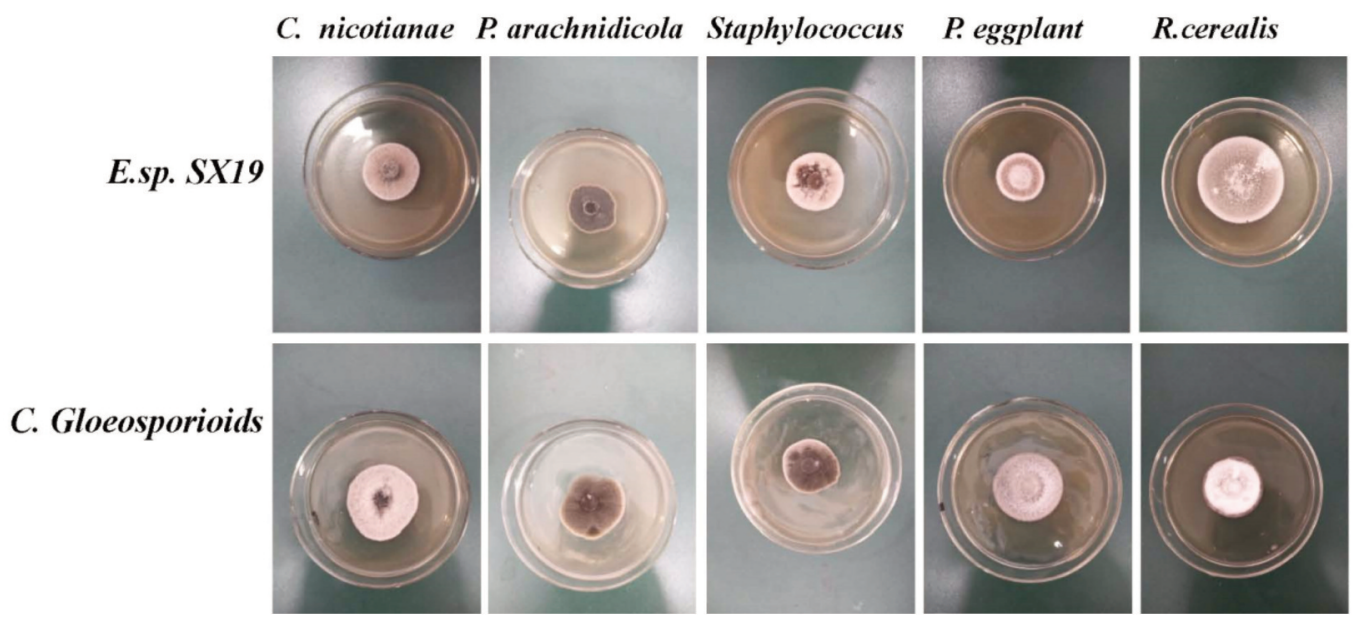

Figure 3. The inhibition rate of E.sp. SX19 and C. Gloeosporioids on 5 plant pathogenic fungi in vitro. The colony diameters of E.sp. SX19 (upper panel) and C. Gloeosporioids (lower panel) under the challenges of five pathogenic fungi, including Cercospora nicotianae, Phoma arachnidicola, Staphylococcus, Phytophthora eggplant, and Rhizoctonia cerealis, were measured. The inhibition rates for each treatment were calculated with the formula: (control plate colony diameter - fermentation broth colony diameter)/control plate colony diameter $\times 100 \%$. 


\section{Acknowledgements}

This project was supported by grants from the Natural Science Foundation of Anhui Province (2008085MH289), National \& Local Joint Engineering Research Center of High-throughput Drug Screening Technology (G20181001), Anhui Province College Excellent Young Talents Fund (gxyqZD2019035) and Key Research and Development Project of Anhui Province (1704a0802145).

\section{References}

1 Tan RX and Zou WX: Endophytes: a rich source of functional metabolites. Nat Prod Rep 18(4): 448-459, 2001. PMID: 11548053. DOI: $10.1039 / \mathrm{b} 1009180$

2 Zhang HW, Song YC and Tan RX: Biology and chemistry of endophytes. Nat Prod Rep 23(5): 753-771, 2006. PMID: 17003908. DOI: 10.1039/b609472b

3 Rodriguez RJ, White JF, Jr., Arnold AE and Redman RS: Fungal endophytes: diversity and functional roles. New Phytol 182(2): 314-330, 2009. PMID: 19236579. DOI: 10.1111/j.14698137.2009.02773.x

4 Venugopalan A and Srivastava S: Endophytes as in vitro production platforms of high value plant secondary metabolites. Biotechnol Adv 33(6 Pt 1): 873-887, 2015. PMID: 26225453. DOI: 10.1016/j.biotechadv.2015.07.004

5 Huang Y, Wang J, Li G, Zheng Z and Su W: Antitumor and antifungal activities in endophytic fungi isolated from pharmaceutical plants Taxus mairei, Cephalataxus fortunei and Torreya grandis. FEMS Immunol Med Microbiol 31(2): 163-167, 2001. PMID: 11549424. DOI: 10.1111/j.1574-695X.2001.tb00513.x

6 Berg G, Krechel A, Ditz M, Sikora RA, Ulrich A and Hallmann $\mathrm{J}$ : Endophytic and ectophytic potato-associated bacterial communities differ in structure and antagonistic function against plant pathogenic fungi. FEMS Microbiol Ecol 51(2): 215-229, 2005. PMID: 16329870. DOI: 10.1016/j.femsec.2004.08.006

7 Paul NC, Kim WK, Woo SK, Park MS and Yu SH: Diversity of endophytic fungi associated with Taraxacum coreanum and their antifungal activity. Mycobiology 34(4): 185-190, 2006. PMID: 24039496. DOI: 10.4489/MYCO.2006.34.4.185

8 Li CH, Zhao MW, Tang CM and Li SP: Population dynamics and identification of endophytic bacteria antagonistic toward plant-pathogenic fungi in cotton root. Microb Ecol 59(2): 344356, 2010. PMID: 19669227. DOI: 10.1007/s00248-009-9570-4

9 Forchetti G, Masciarelli O, Izaguirre MJ, Alemano S, Alvarez D and Abdala G: Endophytic bacteria improve seedling growth of sunflower under water stress, produce salicylic acid, and inhibit growth of pathogenic fungi. Curr Microbiol 61(6): 485-493, 2010. PMID: 20383767. DOI: 10.1007/s00284-010-9642-1

10 Zhang X, Li G, Ma J, Zeng Y, Ma W and Zhao P: Endophytic fungus Trichothecium roseum LZ93 antagonizing pathogenic fungi in vitro and its secondary metabolites. J Microbiol 48(6): 784-790, 2010. PMID: 21221935. DOI: 10.1007/s12275-010-0173-z

11 Johann S, Rosa LH, Rosa CA, Perez P, Cisalpino PS, Zani CL and Cota BB: Antifungal activity of altenusin isolated from the endophytic fungus Alternaria sp. against the pathogenic fungus Paracoccidioides brasiliensis. Rev Iberoam Micol 29(4): 205209, 2012. PMID: 22366718. DOI: 10.1016/j.riam.2012.02.002

12 Qian Y, Yu H, He D, Yang H, Wang W, Wan X and Wang L: Biosynthesis of silver nanoparticles by the endophytic fungus
Epicoccum nigrum and their activity against pathogenic fungi. Bioprocess Biosyst Eng 36(11): 1613-1619, 2013. PMID: 23463299. DOI: 10.1007/s00449-013-0937-z

13 Bernardi-Wenzel J, Garcia A, Azevedo JL and Pamphile JA: Molecular characterization by amplified ribosomal DNA restriction analysis and antimicrobial potential of endophytic fungi isolated from Luehea divaricata (Malvaceae) against plant pathogenic fungi and pathogenic bacteria. Genet Mol Res 12(4): 5072-5084, 2013. PMID: 24301768. DOI: 10.4238/2013.October.29.2

14 Kumar S and Kaushik N: Endophytic fungi isolated from oilseed crop Jatropha curcas produces oil and exhibit antifungal activity. PLoS One 8(2): e56202, 2013. PMID: 23409154. DOI: 10.1371/journal.pone.0056202

15 Mousa WK, Schwan A, Davidson J, Strange P, Liu H, Zhou T, Auzanneau FI and Raizada MN: An endophytic fungus isolated from finger millet (Eleusine coracana) produces anti-fungal natural products. Front Microbiol 6: 1157, 2015. PMID: 26539183. DOI: $10.3389 /$ fmicb.2015.01157

16 Ibrahim M, Kaushik N, Sowemimo A, Chhipa H, Koekemoer T, van de Venter $M$ and Odukoya OA: Antifungal and antiproliferative activities of endophytic fungi isolated from the leaves of Markhamia tomentosa. Pharm Biol 55(1): 590-595, 2017. PMID: 27937112. DOI: 10.1080/13880209.2016.1263671

17 Erfandoust R, Habibipour R and Soltani J: Antifungal activity of endophytic fungi from Cupressaceae against human pathogenic Aspergillus fumigatus and Aspergillus niger. J Mycol Med 30(3): 100987, 2020. PMID: 32499133. DOI: 10.1016/j.mycmed. 2020.100987

18 He Y, Cao Y, Xiang Y, Hu F, Tang F, Zhang Y, Albashari AA, Xing Z, Luo L, Sun Y, Huang Q, Ye Q and Zhang K: An evaluation of norspermidine on anti-fungal effect on mature Candida albicans biofilms and angiogenesis potential of dental pulp stem cells. Front Bioeng Biotechnol 8: 948, 2020. PMID: 32903416. DOI: 10.3389/fbioe.2020.00948

19 Xiao Y, Li HX, Li C, Wang JX, Li J, Wang MH and Ye YH: Antifungal screening of endophytic fungi from Ginkgo biloba for discovery of potent anti-phytopathogenic fungicides. FEMS Microbiol Lett 339(2): 130-136, 2013. PMID: 23240805. DOI: 10.1111/1574-6968.12065

20 Soltani J and Hosseyni Moghaddam MS: Antiproliferative, antifungal, and antibacterial activities of endophytic alternaria species from Cupressaceae. Curr Microbiol 69(3): 349-356, 2014. PMID: 24801337. DOI: 10.1007/s00284-014-0594-8

21 Sanchez-Fernandez RE, Sanchez-Fuentes R, Rangel-Sanchez H, Hernandez-Ortega S, Lopez-Cortes JG and Macias-Rubalcava ML: Antifungal and antioomycete activities and modes of action of isobenzofuranones isolated from the endophytic fungus Hypoxylon anthochroum strain Gseg1. Pestic Biochem Physiol 169: 104670, 2020. PMID: 32828376. DOI: 10.1016/j.pestbp. 2020.104670

22 Zhai X, Jia M, Chen L, Zheng CJ, Rahman K, Han T and Qin LP: The regulatory mechanism of fungal elicitor-induced secondary metabolite biosynthesis in medical plants. Crit Rev Microbiol 43(2): 238-261, 2017. PMID: 27936989. DOI: 10.1080/1040841X.2016.1201041

23 Jiang Y, Wang L, Lu S, Xue Y, Wei X, Lu J and Zhang Y: Transcriptome sequencing of Salvia miltiorrhiza after infection by its endophytic fungi and identification of genes related to tanshinone biosynthesis. Pharm Biol 57(1): 760-769, 2019. PMID: 31694427. DOI: 10.1080/13880209.2019.1680706 
24 Dong XJ, Guo LF, Yao R, Xue SY and Li F: Relationship between regulation effect of Salvia miltiorrhiza on AQP2 in kidney and promoting blood circulation and diuresis. Zhongguo Zhong Yao Za Zhi 39(16): 3162-3165, 2014. PMID: 25509307. DOI: $10.4268 / \mathrm{cjcmm} 20141631$

$25 \mathrm{Chen} \mathrm{H}$ and Chen F: Effect of methyl jasmonate and salicic acid on cell growth and cryptotanshinone formation in Ti transformed Salvia miltiorrhiza cell suspension cultures. Biotechnol Lett 21: 803-807, 1999. DOI: 10.1023/A:1005551911450

26 Liu AH, Guo H, Ye M, Lin YH, Sun JH, Xu M and Guo DA: Detection, characterization and identification of phenolic acids in Danshen using high-performance liquid chromatography with diode array detection and electrospray ionization mass spectrometry. J Chromatogr A 1161(1-2): 170-182, 2007. PMID: 17574558. DOI: $10.1016 /$ j.chroma.2007.05.081

27 Li MH, Peng Y and Xiao PG: Distribution of tanshinones in the genus Salvia (family Lamiaceae) from China and its systematic significance. J Syst Evol 48(2): 118-122, 2010. DOI: 10.1111/1759-6831.2010.00070.x

28 Chen YH, Lin SJ, Ku HH, Shiao MS, Lin FY, Chen JW and Chen YL: Salvianolic acid B attenuates VCAM-1 and ICAM-1 expression in TNF-alpha-treated human aortic endothelial cells. J Cell Biochem 82(3): 512-521, 2001. PMID: 11500927. DOI: $10.1002 /$ jcb. 1176

29 Zhang H, Liu YY, Jiang Q, Li KR, Zhao YX, Cao C and Yao J: Salvianolic acid A protects RPE cells against oxidative stress through activation of Nrf2/HO-1 signaling. Free Radic Biol Med 69: 219-228, 2014. PMID: 24486344. DOI: 10.1016/j.freerad biomed.2014.01.025

30 Zhao T, Chang L, Zhang B, Lu M, Wang X, Orgah JO, Wang Y, Tian X, Yang J, Fan G, Zhang B and Zhu Y: Specific combination of Salvianolic acids as core active ingredients of Danhong injection for treatment of arterial thrombosis and its derived dry gangrene. Front Pharmacol 8: 361, 2017. PMID: 28659797. DOI: $10.3389 /$ fphar.2017.00361

31 Sun B, Li C, Zuo L and Liu P: Protection of SAL B with H9C2 cells. Pharm Biol 54(5): 889-895, 2016. PMID: 26705025. DOI: 10.3109/13880209.2015.1089911

32 Yang B, Zheng C, Yu H, Zhang R, Zhao C and Cai S: Cardioprotective effects of salvianolic acid B on oxygen and glucose deprivation (OGD)-treated H9c2 cells. Artif Cells Nanomed Biotechnol 47(1): 2274-2281, 2019. PMID: 31184214. DOI: 10.1080/21691401.2019.1621885

33 Yang W, Ju JH, Jeon MJ, Han X and Shin I: Danshen (Salvia miltiorrhiza) extract inhibits proliferation of breast cancer cells via modulation of Akt activity and p27 level. Phytother Res 24(2): 198-204, 2010. PMID: 19610045. DOI: 10.1002/ptr.2945

34 Jung I, Kim H, Moon S, Lee H and Kim B: Overview of salvia miltiorrhiza as a potential therapeutic agent for various diseases: An update on efficacy and mechanisms of action. Antioxidants (Basel) 9(9): E857, 2020. PMID: 32933217. DOI: 10.3390/antiox 9090857
35 Sun J, Huang SH, Tan BK, Whiteman M, Zhu YC, Wu YJ, Ng Y, Duan W and Zhu YZ: Effects of purified herbal extract of Salvia miltiorrhiza on ischemic rat myocardium after acute myocardial infarction. Life Sci 76(24): 2849-2860, 2005. PMID: 15808885. DOI: $10.1016 /$ j.lfs.2004.11.016

36 Wang L, Ma R, Liu C, Liu H, Zhu R, Guo S, Tang M, Li Y, Niu J, Fu M, Gao S and Zhang D: Salvia miltiorrhiza: A potential red light to the development of cardiovascular diseases. Curr Pharm Des 23(7): 1077-1097, 2017. PMID: 27748194. DOI: 10.2174/1381612822666161010105242

$37 \mathrm{Li} \mathrm{ZM}$, Xu SW and Liu PQ: Salvia miltiorrhiza Burge (Danshen): a golden herbal medicine in cardiovascular therapeutics. Acta Pharmacol Sin 39(5): 802-824, 2018. PMID: 29698387. DOI: 10.1038/aps.2017.193

38 Zhou Y, Li W, Xu L and Chen L: In Salvia miltiorrhiza, phenolic acids possess protective properties against amyloid beta-induced cytotoxicity, and tanshinones act as acetylcholinesterase inhibitors. Environ Toxicol Pharmacol 31(3): 443-452, 2011. PMID: 21787715. DOI: 10.1016/j.etap.2011.02.006

39 Zhang XZ, Qian SS, Zhang YJ and Wang RQ: Salvia miltiorrhiza: A source for anti-Alzheimer's disease drugs. Pharm Biol 54(1): 1824, 2016. PMID: 25857808. DOI: 10.3109/13880209.2015.1027408

40 Lee TY, Chang HH, Wang GJ, Chiu JH, Yang YY and Lin HC: Water-soluble extract of Salvia miltiorrhiza ameliorates carbon tetrachloride-mediated hepatic apoptosis in rats. J Pharm Pharmacol 58(5): 659-665, 2006. PMID: 16640835. DOI: $10.1211 /$ jpp.58.5.0011

$41 \mathrm{Li}$ YL, Xin XM, Chang ZY, Shi RJ, Miao ZM, Ding J and Hao GP: The endophytic fungi of Salvia miltiorrhiza Bge.f. alba are a potential source of natural antioxidants. Bot Stud 56(1): 5, 2015. PMID: 28510814. DOI: 10.1186/s40529-015-0086-6

42 Li X, Zhai X, Shu Z, Dong R, Ming Q, Qin L and Zheng C: Phoma glomerata D14: An endophytic fungus from Salvia miltiorrhiza that produces salvianolic acid C. Curr Microbiol 73(1): 31-37, 2016. PMID: 26979309. DOI: 10.1007/s00284016-1023-y

43 Wei JC: Identification of fungus handbook. Shanghai: Science Press, 1979.

44 Zhang PL, Wang GK and Yu Y: Isolation and identification of endophytic fungus from Paeonia ostii and study their diversity in different growth periods. J Anhui Univ Chinese Med 35: 7882, 2016. DOI: 10.3969/j.issn.2095-7246.2016.05.023

Received October 8, 2020

Revised October 21, 2020

Accepted October 27, 2020 